PESQUIMAT Revista de la Fac. CC. MM. de la

Universidad Nacional Mayor de San Marcos

Vol. IX N², pp 1 - 15, LIMA - PERÚ. Diciembre 2006

\title{
A Transmission Problem with Nonlinear Damping and Source Terms
}

\author{
Eugenio Cabanillas Lapa ${ }^{1}$ \\ cleugenio@yahoo.com \\ B. Godoy Torres ${ }^{1}$ \\ dontgise@hotmail.com
}

\author{
Z.Huaringa Segura ${ }^{1}$ \\ zhuaringas@yahoo.es \\ V. Yauri Luque ${ }^{1}$ \\ vyauri@unmsm.edu.pe
}

\section{Resumen}

In this article we study the wave propagation over materials consisting of two components: one component is simple elastic while the other has a nonlinear internal damping with elastic coefficients dependent on time; both components having source terms. By using the potential well method we obtain the global existence, we also show that the energy of the system decays uniformly to zero.

Keywords: stability, transmission problem, internal damping, source terms.

\section{Resumen}

En este trabajo, estudiamos la propagación de ondas sobre materiales que consisten de dos componentes: un componente es simple elástico, mientras que el otro tiene un damping interno no lineal con coeficientes elásticos dependientes del tiempo; ambos componentes tienen términos fuente. Usando el método del potencial, obtenemos la existencia global, también mostramos que la energía del sistema decae uniformemente a cero.

Palabras Clave: Estabilidad, problema de transmisión, damping interno, término fuente.

\section{Introduction}

The main purpose of this work is to study the asymptotic behavior of solutions of the following nonlinear transmission problem

\footnotetext{
${ }^{1}$ UNMSM, Facultad de Ciencias Matemáticas, Lima - Perú.
} 


$$
\begin{array}{|lc}
\left.\rho_{1} u_{t t}-b u_{x x}=\mu_{1} f_{1}(u), \text { in }\right] 0, L_{0}\left[\times \mathbb{R}^{+}\right. \\
\rho_{2} v_{t t}-a(t) v_{x x}+g\left(v_{t}\right)=\mu_{2} f_{2}(v), & \text { in }] L_{0}, L\left[\times \mathbb{R}^{+}\right. \\
u(x, 0)=0=v(L, t) & , t>0 \\
u\left(L_{0}, t\right)=v\left(L_{0}, t\right) & , t>0 \\
b u_{x}\left(L_{0}, t\right)=a(t) v_{x}\left(L_{0}, t\right) & , t>0 \\
u(x, 0)=u^{0}(x), \quad u_{t}(x, 0)=u^{1}(x), & , x \in] 0, L_{0}[ \\
v(x, 0)=v^{0}(x), \quad v_{t}(x, 0)=v^{1}(x) & , x \epsilon] L_{0}, L[
\end{array}
$$

where $\rho_{1}, \rho_{2}$ are different densities of the material, $\mu_{i} \in \mathbb{R}, i=1,2, \quad b>0, \quad g$ is a nondecreasing $C^{1}$ function, $a$ is a elastic coefficient dependent on time and $f_{i}$ is a function like $-|u|^{p_{i}-1} u, \quad p_{i} \geq 1, \quad i=1,2$.

Transmission problem or diffraction problems arise in several applications in physics and biology. The stability of conservative system by means of a internal damping has been studied by many authors (see [ $1-7]$, among others). For the transmission problem there exists several works about controllability and stabilization by mens of feedback fuctions on a part of the boundary (see [8], [11], [12]).

When the coefficients dependent on time and $f_{i}(s) s \geq 0, \mu_{i}=-1$, Muñoz Rivera and Cabanillas L.[14] showed that solutions converge to zero exponentially. In our case we have $\mu_{i} \in \mathbb{R},\left|f_{i}(s)\right| \leq|s|^{p_{i}}, \forall s \in \mathbb{R}$, with non linear damping $g$.

The first part of this paper is to study the global existence of regular and weak solution to problem $(1,1)-(1,7)$, here we have there some technical difficulties that we need over come because of the coefficient dependent on time and the source term. Semigroup arguments are not suitable for finding solutions to $(1,1)-(1,7)$; the method in $[8]$ does not seen to be directly applicable to the function $f_{i}$, therefore, we make use of a Galerkin approximation and the potential well method.

The second part is to give energy decay estimates of the solution of $(1,1)-(1,7)$ for a general non-linear damping $g$. We found that rate of decay of the solutions depend on behavior of the dissipative term in a neighborhood of zero, that is for a linear dissipation we obtain exponential decay while for a polynomial dissipation we obtain polynomial decay.

Our paper is organized as follows: In section 2, we present the notations and statement of results. In section 3 , we prove solvability of $(1,1)-(1,7)$, while section 4 deals with the asymptotic behavior of the solutions obtained in section 3 .

\section{Notations and statement of results}

We denote

$$
(w, z)=\int_{I} w(x, z(x)) d x, \quad|z|^{2}=\int_{I}|z(x)|^{2} d x
$$


where $I=] 0, L_{0}[$ or $] L_{0}, L\left[\right.$ for $u^{\prime} s$ and $v^{\prime} s$ respectively. Assume that

(A1) We take $f_{i} \in C^{1}(\mathbb{R}), i=1,2, \quad f_{i}(0)=0$

$$
\left|f_{i}^{\prime}(s)\right| \leq|s|^{p_{i}-1} \quad 1 \leq p_{1}, p_{2}<\infty
$$

and without less of generality, we assume $p_{1} \geq p_{2}$.

$$
F_{i}(s)=\mu_{i} \int_{0}^{s} f_{i}(\xi) d \xi
$$

(A2) Let $g: \mathbb{R} \rightarrow \mathbb{R}$ be a nondecreasing $C^{1}$ function such that

$$
g(s) . s>0 \quad \text { for all } s \neq 0,
$$

and there exist $c_{i}>0, i=1,2,3,4$ such that

$$
\left\{\begin{array}{l}
c_{1}|s|^{p} \leq|g(s)| \leq c_{2}|s|^{1 / p} ; \text { if }|s| \leq 1 \\
c_{3}|s| \leq|g(s)| \leq c_{4}|s|, \quad \text { if }|s|>1
\end{array}\right.
$$

where $p \geq 1$

(A3) $a \in W_{\text {Loc }}^{1,1}(0, \infty), \quad a(t) \geq a_{0}>0$, for some $a_{0}>0$

By $V$ we denote the Hilbert Space

$$
V=\left\{(w, z) \in H^{1}\left(0, L_{0}\right) \times H^{1}\left(L_{0}, L\right): w(0)=z(L)=0 ; w\left(L_{0}\right)=z\left(L_{0}\right)\right\}
$$

By $E_{1}$ and $E_{2}$ we denote the first order energy associated to each equation

$$
\begin{aligned}
& E_{1}(t, u)=\frac{1}{2} \rho_{1}\left|u_{t}(t)\right|^{2}+\frac{b}{2}\left|u_{x}(t)\right|^{2}-\int_{0}^{L_{0}} F_{1}(u) d x \\
& E_{2}(t, v)=\frac{1}{2} \rho_{2}\left|v_{t}(t)\right|^{2}+\frac{a(t)}{2}\left|v_{x}(t)\right|^{2}-\int_{L_{0}}^{L} F_{2}(v) d x \\
& J_{1}(u)=\frac{1}{2} b\left|u_{x}\right|^{2}-\frac{\mu_{1}}{p_{1}+1}|u|_{p_{1}+1}^{p_{1}+1} \\
& J_{2}(v)=\frac{1}{2} a_{0}\left|v_{x}\right|^{2}-\frac{\mu_{2}}{p_{2}+1}|v|_{p_{2}+1}^{p_{2}+1} \\
& I(u, v)=b\left|u_{x}\right|^{2}+a_{0}\left|v_{x}\right|^{2}-\mu_{1}|u|_{p_{1}+1}^{p_{1}+1}-\mu_{2}|v|_{p_{2}+1}^{p_{2}+1} \\
& E(t) \equiv E(t, u, v)=E_{1}(t, u)+E_{2}(t, v)
\end{aligned}
$$


and define the stable set as $W=\{(u, v) \in V: I(u, v)>0\} \cup\{0\}$

In order to show the decay property we will need the following lemma

Lemma 2.1 Let $E: \mathbb{R}_{+} \rightarrow \mathbb{R}_{+}$be a nonincreasing function, and assume that there exist two constant $p>0$ and $c>0$ such that

$$
\int_{s}^{+\infty} E^{\frac{p+1}{2}}(t) d t \leq c E(s) \quad 0 \leq s<+\infty
$$

The, we have

$$
\begin{aligned}
& E(t) \leq c E(0)(1+t)^{\frac{-2}{p-1}}, \text { for all } t \geq 0 \text { and } p>1 \\
& E(t) \leq c E(0) e^{1-w t}, \quad \text { for all } t \geq 0 \text { if } p=1
\end{aligned}
$$

where $c$ and $w$ are positive constants.

\section{Existence and uniqueness of solutions}

We begin this section defining what we mean by weak solution to the system $(1,1)-(1,7)$.

Definition 3.1 We say that couple $\{u, v\}$ is a weak solution of $(1,1)-(1,7)$ when

$$
\{u, v\} \in L^{\infty}(0, T ; V) \cap W^{1, \infty}\left(0, T, L^{2}\left(0, L_{0}\right) \times L^{2}\left(L_{0}, L\right)\right)
$$

and satisfies

$$
\begin{aligned}
& -\rho_{1} \int_{0}^{L_{0}} u^{1}(x) \varphi(x, 0) d x-\rho_{2} \int_{L_{0}}^{L} v^{1}(x) \psi(x, 0) d x-\rho_{1} \int_{0}^{T} \int_{0}^{L_{0}} u_{t} \varphi_{t} d x d t \\
& -\rho_{2} \int_{0}^{T} \int_{L_{0}}^{L} v_{t} \psi_{t} d x d t+b \int_{0}^{T} \int_{0}^{L_{0}} u_{x} \varphi_{x} d x d t+\int_{0}^{T} \int_{0}^{L_{0}} f_{1}(u) \varphi d x d t \\
& +\int_{0}^{T} a(t) \int_{L_{0}}^{L} v_{x} \psi_{x} d x d t+\int_{0}^{T} \int_{L_{0}}^{L} f_{2}(v) \psi d x d t+\int_{0}^{T} \int_{L_{0}}^{L} g\left(v_{t}\right) \psi d x d t=0
\end{aligned}
$$

for any $\{\varphi, \psi\} \in C^{2}(0, T ; V)$ such that,

$$
\varphi(T)=\varphi_{t}(T)=\psi(T)=\psi_{t}(T)=0 .
$$

In order to show the existence of strong solutions are need a regularity result for the elliptic 
system associated to the problem $(1,1)-(1,7)$ whose proof can be obtained with little modifications in the book O.A. Ladyzhenkaya and N. N. Ural'tseva ([14], theorem 16.2)

Lemma 3.2 For any given functions $F \in L^{2}\left(0, L_{0}\right), G \in L^{2}\left(L_{0}, L\right)$ there exists only solution $\{u, v\}$ of

$$
\mid \begin{aligned}
& \left.-b u_{x x}=F \text { in }\right] 0, L_{0}[ \\
& \left.-a(t) v_{x x}=G \text { in }\right] L_{0}, L[ \\
& u(0)=v(L)=0 \\
& u\left(L_{0}\right)=v\left(L_{0}\right), \quad b u_{x}\left(L_{0}\right)=a(t) v_{x}\left(L_{0}\right)
\end{aligned}
$$

with $t$ a fixed value in $[0, T]$ satisfying $u \in H^{2}\left(0, L_{0}\right)$ and $v \in H^{2}\left(L_{0}, L\right)$

Now we are in a position to state the global existence results.

Theorem 3.3 Suppose that assumptions (A1) - (A3) holds.

If $\left\{u^{0}, v^{0}\right\} \in W,\left\{u^{1}, v^{1}\right\} \in L^{2}\left(0, L_{0}\right) \times L^{2}\left(L_{0}, L\right)$ and

$$
\begin{aligned}
& \operatorname{máx}\left\{\left|\mu_{1}\right| b^{-1} c_{x}^{p_{1}+1}\left[\frac{2}{b} \frac{p_{2}+1}{p_{2}-1} E(0) \exp \int_{0}^{T} \frac{\left|a^{\prime}(s)\right|}{a(s)} d s\right]^{\frac{p_{1}-1}{2}},\right. \\
& \left.\left|\mu_{2}\right| a_{0}^{-1} c_{x}^{p_{2}+1}\left[\frac{2}{a_{0}} \frac{p_{2}+1}{p_{2}-1} E(0) \exp \int_{0}^{T} \frac{\left|a^{\prime}(s)\right|}{a(s)} d s\right]^{\frac{p_{2}-1}{2}}\right\}<1
\end{aligned}
$$

then there exists a unique local solution of $(1,1)-(1,7)$ satisfying

$$
\{u, v\} \in C(0, T ; V) \cap C^{1}\left(0, T ; L^{2}\left(0, L_{0}\right) \times L^{2}\left(L_{0}, L\right)\right)
$$

In addition, if $\left\{u^{0}, v^{0}\right\} \in W \cap\left(H^{2}\left(0, L_{0}\right) \times H^{2}\left(L_{0}, L\right)\right),\left\{u^{1}, v^{1}\right\} \in V$ and $(3,1)$ holds, verifying the compatibility condition

$$
b u_{x}^{0}\left(L_{0}\right)=a(0) v_{x}^{0}\left(L_{0}\right)
$$

then there exists a strong solution $\{u, v\}$ satisfying

$$
\begin{aligned}
\{u, v\} \in & C\left(0, T ; H^{2}\left(0, L_{0}\right) \times H^{2}\left(L_{0}, L\right)\right) \cap C^{1}(0, T ; V) \cap \\
& C^{2}\left(0, T ; L^{2}\left(0, L_{0}\right) \times L^{2}\left(L_{0}, L\right)\right)
\end{aligned}
$$

Proof We employ the Galerkin Method to construct a solution.

Let $\left\{\left\{\varphi^{i}, \psi^{i}\right\}, \quad i=1,2, \ldots\right\}$ be a basis to $V$. We construct approximate solution

$$
\left\{u^{m}(t), v^{m}(t)\right\}=\sum_{i=1}^{m} h_{i m}(t)\left\{\varphi^{i}, \psi^{i}\right\}
$$


which is determinate by the ordinary differential equations

$$
\begin{aligned}
& \rho_{1}\left(u_{t t}^{m}, \varphi^{i}\right)+b\left(u_{x}^{m}, \varphi_{x}^{i}\right)+\left(f_{1}\left(u^{m}\right), \varphi^{i}\right)+\rho_{2}\left(v_{t t}^{m}, \psi^{i}\right)+ \\
& a(t)\left(v_{x}^{m}, \psi_{x}^{i}\right)+\left(g\left(v_{t}^{m}\right), \psi^{i}\right)+\left(f_{2}\left(v^{m}\right), \psi^{i}\right)=0
\end{aligned}
$$

where $i=1,2,3 \ldots$

with the initial conditions

$$
\left\{u^{m}(0), v^{m}(0)\right\}=\left\{u^{0}, v^{0}\right\}, \quad\left\{u_{t}^{m}(0), v_{t}^{m}(0)\right\}=\left\{u^{1}, v^{1}\right\}
$$

By standard methods in differential equations we prove the existence of solutions to $(3,3)$ $(3,4)$ on some interval $\left[0, T_{m}\left[, \quad 0<T_{m} \leq \infty\right.\right.$. In order to extend the solution of $(3,3)-(3,4)$ to the whole interval $[0, \infty[$ we need the priori estimate below.

Weak solutions. Multiplying $(3,3)$ by $h_{i m}^{\prime}(t)$, integrating by parts and summing up on $i$ we get

$$
\frac{d}{d t} E\left(t, u^{m}, v^{m}\right)+\left(g\left(v_{t}^{m}\right), v_{t}^{m}\right) \leq \frac{\left|a^{\prime}(t)\right|}{a(t)} E\left(t, u^{m}, v^{m}\right)
$$

Integrating $(3,5)$ over $] 0, t[$, it hold that

$$
\begin{gathered}
E\left(t, u^{m}, v^{m}\right)+\int_{0}^{t}\left(g\left(v_{t}^{m}(s)\right), v_{t}^{m}(s)\right) d s \\
\leq E\left(0, u^{0}, v^{0}\right)+\int_{0}^{t} \frac{\left|a^{\prime}(s)\right|}{a(s)} E\left(s, u^{m}(s), v^{m}(s)\right)
\end{gathered}
$$

Employing gronwall's lemma, from the last inequality we see that

$$
\begin{aligned}
& E\left(t, u^{m}(t), v^{m}(t)\right)+\int_{0}^{t}\left(g\left(v_{t}^{m}(s)\right), v_{t}^{m}(s)\right) d s \\
& \leq E\left(0, u^{0}, v^{0}\right) \exp \left(\int_{0}^{t} \frac{\left|a^{\prime}(s)\right|}{a(s)} d s\right), 0 \leq t \leq T
\end{aligned}
$$

Now to obtain a priori estimates, we need the following result

Lemma 3.4 Let $\left\{u^{m}(t), v^{m}(t)\right\}$ be the solution of $(3,3)-(3,4)$ with $\left\{u^{0}, v^{0}\right\} \in W \cap V$ and $\left\{u^{1}, v^{1}\right\} \in L^{2}\left(0, L_{0}\right) \times L^{2}\left(L_{0}, L\right)$. 
If

$$
\begin{gathered}
\alpha=\operatorname{máx}\left\{\left|u_{1}\right| b^{-1} c_{x}^{p_{1}+1}\left[\frac{2}{b} \frac{P_{2}+1}{P_{2}-1} E(0) e \times y \int_{0}^{T} \frac{\left|a^{1}(s)\right|}{a(s)} d s\right]^{\frac{P_{1}-1}{2}},\right. \\
\left.\left|\mu_{2}\right| a_{0}^{-1} c_{x}^{P_{2}+1}\left[\frac{2}{a_{0}} \frac{P_{2}+1}{P_{2}-1} E(0) \exp \int_{0}^{T} \frac{\left|a^{1}(s)\right|}{a(s)} d s\right]^{\frac{P_{2}-1}{2}}\right\}<1
\end{gathered}
$$

Then $\{u(t), v(t)\} \in W \cap V$ on $[0, T]$, that is, for all $t \in[0, T]$

$$
I\left(u^{m}(t), v^{m}(t)\right)>0
$$

Proof. Since $I\left(u^{0}, v^{0}\right)>0$ it follows from the continuity of $\left\{u^{m}(t), v^{m}(t)\right\}$ that

$$
I\left(u^{m}(t), v^{m}(t)\right) \geq 0
$$

for some interval near to $t=0$

Let $t_{\text {máx }}>0$ be a maximal time (possibly $t_{\text {máx }}=T_{m}$ ) such that $(3,7)$ holds on $\left[0, t_{\text {máx }}[\right.$. In order to facilitate the notation, we will omit the index $m$ of the solution of the approximate system. Note that

$$
\begin{aligned}
J(u(t), v(t)) \geq & \frac{b}{2}\left|u_{x}\right|^{2}+\frac{a_{0}}{2}\left|v_{x}\right|^{2}-\frac{\mu_{1}}{p_{1}+1}|u|_{p_{1}+1}^{p_{1}+1}-\frac{\mu_{2}}{p_{2}+1}|v|_{p_{2}+1}^{p_{2}+1} \\
= & \frac{1}{p_{2}+1} I(u(t) ; v(t))+\frac{b\left(p_{2}-1\right)}{2\left(p_{2}+1\right)}\left|u_{x}\right|^{2}+\frac{a_{0}\left(p_{2}-1\right)}{2\left(p_{2}+1\right)}\left|v_{x}\right|^{2} \\
& +\frac{\mu_{1}\left(p_{1}-p_{2}\right)}{\left(p_{1}+1\right)\left(p_{2}+1\right)}|u|_{p_{1}+1}^{p_{1}+1} \\
\geq & \frac{p_{2}-1}{2\left(p_{2}+1\right)}\left\{b\left|u_{x}\right|^{2}+a_{0}\left|v_{x}\right|^{2}\right\}, \forall t \in\left[0, t_{\text {máx }}[\right.
\end{aligned}
$$

Consequently, we get

$$
\begin{aligned}
b\left|u_{x}\right|^{2}+a_{0}\left|v_{x}\right|^{2} & \leq \frac{2\left(p_{2}+1\right)}{p_{2}-1} J(u(t), v(t)) \\
& \leq \frac{2\left(p_{2}+1\right)}{p_{2}-1} E(t, u, v) \\
& \leq \frac{2\left(p_{2}+1\right)}{p_{2}-1}\left[E\left(0, u^{0}, v^{0}\right) \exp \int_{0}^{T} \frac{\left|a^{1}(s)\right|}{a(s)} d s\right] \text { in }[0, t \text { máx }]
\end{aligned}
$$


It follows from the Sobolev-Poincare inequality and $(3,8)$ that

$$
\begin{aligned}
\mu|v|_{p_{1}+1}^{p_{1}+1} & \leq\left|\mu_{1}\right| c_{x}^{p_{1}+1}\left|u_{x}\right|^{p_{1}+1}=\frac{\left|\mu_{1}\right|}{b} c_{x}^{p_{1}+1}\left|u_{x}\right|^{p_{1}-1}\left(b\left|u_{x}\right|^{2}\right) \\
& \leq \frac{\left|\mu_{1}\right|}{b} c_{x}^{p_{1}+1}\left[\frac{2}{b} \frac{p_{2}+1}{\left(p_{2}-1\right)} E(0) \exp \int_{0}^{T} \frac{\left|a^{1}(s)\right|}{a(s)} d s\right]^{\frac{p_{1}-1}{2}} b\left|u_{x}\right|^{2}
\end{aligned}
$$

Similarly

$$
\begin{gathered}
\mu_{2}|v|_{p_{2}+1}^{p_{2}+1} \leq \frac{\left|\mu_{2}\right|}{a_{0}} c_{x}^{p_{2}+1}\left[\frac{2}{a_{o}} \frac{p_{2}+1}{p_{2}-1} E(0) \exp \int_{0}^{T} \frac{\left|a^{1}(s)\right|}{a(s)} d s\right]^{\frac{p_{2}-1}{2}} a_{0}\left|v_{x}\right|^{2} \\
<a_{0}\left|v_{x}\right|^{2}
\end{gathered}
$$

this from $(3,9)$ and $(3,10)$ we obtain

$$
\mu_{1}|u|_{p_{1}+1}^{p_{1}+1}+\mu_{2}+|v|_{p_{2}+1}^{p_{2}+1}<b\left|u_{x}\right|^{2}+a_{0}\left|v_{x}\right|^{2}
$$

Therefore we get $I(u(t) ; v(t))>0$ on $\left[0, t\right.$ máx [. This implies that we can take $t_{\text {máx }}=T_{m}$. This completes the proof of lemma 3.4.

Using lemma 3.4., we can deduce a priori estimate for $\{u(t), v(t)\}$. Lemma 3.4 implies that

$$
\begin{aligned}
& E(t, u(t), v(t))=\frac{1}{2}\left|u_{t}(t)\right|^{2}+\frac{1}{2}\left|v_{t}(t)\right|^{2}+J(u(t), v(t)) \\
\geq & \frac{1}{2}\left|u_{t}(t)\right|^{2}+\frac{1}{2}\left|v_{t}(t)\right|^{2}+\frac{1}{p_{2}+1} I(u(t), v(t))+ \\
& +\frac{b\left(p_{2}-1\right)}{2\left(p_{2}+1\right)}\left|u_{x}\right|^{2}+\frac{a_{0}\left(p_{2}-1\right)}{2\left(p_{2}+1\right)}\left|v_{x}\right|^{2}+\frac{\mu_{1}\left(p_{1}-p_{2}\right)}{\left(p_{1}+1\right)\left(p_{2}+1\right)}|u|_{p_{1}+1}^{p_{1}+1} \\
\geq & \frac{1}{2}\left|u_{t}(t)\right|^{2}+\frac{1}{2}\left|v_{t}(t)\right|^{2}+\frac{p_{2}-1}{2\left(p_{2}+1\right)}\left(b\left|u_{x}\right|^{2}+a_{0}\left|v_{x}\right|^{2}\right)
\end{aligned}
$$

From $(3,6)$ and $(3,12)$, we get

$$
\begin{aligned}
& \frac{1}{2}\left|u_{t}(t)\right|^{2}+\frac{1}{2}\left|v_{t}(t)\right|^{2}+\frac{p_{2}-1}{2\left(p_{2}+1\right)}\left(b\left|u_{x}\right|^{2}+a_{0}\left|v_{x}\right|^{2}\right)+\int_{0}^{t}\left(g\left(v_{t}(s)\right), v_{t}(s)\right) d s \\
\leq & E\left(0, u^{0}, v^{0}\right) \exp \left(\int_{0}^{T} \frac{\left|a^{\prime}(s)\right|}{a(s)} d s\right) \leq L_{1},
\end{aligned}
$$

where $L_{1}$ is a positive constant independent of $m \in \mathbb{N}$ and $t \in[0, T]$.

Thus, we deduce that

$$
\left\{u^{m}, v^{m}\right\} \text { is bounded in } L^{\infty}(0, T ; V)
$$




$$
\left\{u_{t}^{m}, v_{t}^{m}\right\} \text { is bounded in } L^{\infty}\left(0, T ; L^{2}\left(0, L_{0}\right) \times L^{2}\left(L_{0}, L\right)\right)
$$

wich imply that

$$
\begin{aligned}
& \left\{u^{m}, v^{m}\right\} \rightarrow\{u, v\} \text { weakly *in } L^{\infty}(0, T ; V) \\
& \left\{u_{t}^{m}, v_{t}^{m}\right\} \rightarrow\left\{u_{t}, v_{t}\right\} \text { weakly * in } L^{\infty}\left(0, T ; L^{2}\left(0, L_{0}\right) \times L^{2}\left(L_{0}, L\right)\right)
\end{aligned}
$$

Using Aubin-Lions compactness lemma we have

$$
\left\{u^{m}, v^{m}\right\} \rightarrow\{u, v\} \text { strongly in } L^{2}\left(0, T ; L^{2}\left(0, L_{0}\right) \times L^{2}\left(L_{0}, L\right)\right)
$$

and consequently

$$
\begin{aligned}
& \left.\left.\left.\left.u^{m} \rightarrow u \text { a.e in }\right] 0, L_{0}\right] \text { and } f_{1}\left(u^{m}\right) \rightarrow f_{1}(u) \text { a.e. in }\right] 0, L_{0}\right] \\
& \left.\left.\left.\left.v^{m} \rightarrow v \text { a.e in }\right] L_{0}, L\right] \text { and } f_{2}\left(v^{m}\right) \rightarrow f_{2}(v) \text { a.e. in }\right] L_{0}, L\right]
\end{aligned}
$$

Besides, from the growth condition in $(A 1)$ we have that

$$
\begin{aligned}
& f_{1}\left(u^{m}\right) \text { is bounded in } L^{2}\left(0, T ; L^{2}\left(0, L_{0}\right)\right) \\
& f_{2}\left(v^{m}\right) \text { is bounded in } L^{2}\left(0, T ; L^{2}\left(L_{0}, L\right)\right)
\end{aligned}
$$

and therefore

$$
\left\{f_{1}\left(u^{m}\right), f_{2}\left(v^{m}\right)\right\} \rightarrow\left\{f_{1}(u), f_{2}(v)\right\} \text { in } L^{2}\left(0, T ; L^{2}\left(0, L_{0}\right) \times L^{2}\left(L_{0}, L\right)\right)
$$

Now, we note that from $(3,13)$ and the assumption $(A 2)$, we get

$$
\int_{0}^{t}\left|g\left(v_{t}^{m}(s)\right)\right|^{2} d s \leq L
$$

where $L$ is a positive constant independent of $m$ and $t$.

So, we can take a subsequence, still denote by $\left(v^{m}\right)$ such that

$$
g\left(v_{t}^{m}\right) \rightarrow \chi \text { weakly in } L^{2}(] L_{0}, L[\times] 0, T[)
$$

Returning to $(3,8)$ and using standard argument we can show, from the convergence above that

$$
\begin{aligned}
\rho_{1} u_{t t}-b u_{x x} & =\mu_{1} f_{1}(u) \text { in } L^{2}\left(0, T ; H^{-1}\left(0, L_{0}\right)\right) \\
\rho_{2} v_{t t}-a(t) v_{x x}+\chi & =\mu_{2} f_{2}(v) \text { in } L^{2}\left(0, T ; H^{-1}\left(L_{0}, L\right)\right)
\end{aligned}
$$

Our goal is to prove that

$$
\chi=g\left(v_{t}\right)
$$

but this relation follows from a standard theory of monotone and semicontinuous operators (cf. [09]) and the proof is omitted. 
Therefore, $\{u, v\}$ satisfies $(1,1)-(1,5)$.

Regularity of solutions: To get the regularity result, we take a basis

$$
B=\left\{\left\{\varphi^{i}, \psi^{i}\right\}, i \in \mathbb{N}\right\}
$$

such that

$$
\left\{u^{0}, v^{0}\right\},\left\{u^{1}, v^{1}\right\} \in \operatorname{span}\left\{\left\{\varphi^{0}, \psi^{0}\right\},\left\{\varphi^{1}, \psi^{1}\right\}\right\}
$$

Let us differenciate the approximate equation and multiply by $h_{i m}^{\prime}(t)$. Using a similar argument as before, we obtain

$$
\begin{aligned}
& \frac{d}{d t} E_{2}(t, u, v)+\int_{L_{0}}^{L} g^{\prime}\left(v_{t}^{m}(x, t)\right)\left(v_{t t}^{m}(x, t)\right)^{2} d x \\
= & \mu_{1}\left(f_{1}^{\prime}\left(u_{m}\right) u_{t}^{m}, u_{t t}^{m}\right)+\mu_{2}\left(f_{2}^{\prime}\left(v_{m}\right) v_{t}^{m}, v_{t t}^{m}\right) \\
& -a_{t}(t)\left(v_{x}^{m}, v_{x t t}^{m}\right)+\frac{1}{2} a_{t}(t)\left|v_{t t}\right|^{2}
\end{aligned}
$$

where

$$
E_{2}(t, u, v)=\frac{\rho_{1}}{2}\left|u_{t t}\right|^{2}+\frac{b}{2}\left|u_{x t}\right|^{2}+\frac{\rho_{2}}{2}\left|v_{t t}\right|^{2}+\frac{a(t)}{2}\left|v_{x t}\right|^{2}
$$

Note that

$$
\begin{aligned}
& -a_{t}\left(v_{x}^{m}, v_{x t t}^{m}\right)=-\left(a_{t}\left(v_{x}^{m}, v_{x t}^{m}\right)\right)_{t}+a_{t t}\left(v_{x}^{m}, v_{x t}^{m}\right)+a_{t}\left|v_{x t}^{m}\right|^{2} \\
& E_{2}\left(0, u^{m}, v^{m}\right) \text { in bounded, because on choice of the basis. }
\end{aligned}
$$

Now, from the growth condition $(A 1)$ and the Sobolev imbedding we have

$$
\int_{0}^{L_{0}} f_{1}^{\prime}\left(u^{m}\right) u_{t}^{m} u_{t t}^{m} d x \leq c\left[\int_{0}^{L_{0}}\left|u_{x}^{m}\right|^{2} d x\right]^{\frac{p_{1}-1}{2}}\left|u_{x t}^{m}\right|\left|u_{t t}^{m}\right|
$$

and similarly

$$
\int_{L_{0}}^{L} f_{2}^{\prime}\left(v^{m}\right) v_{t}^{m} v_{t t}^{m} d x \leq c\left[\int_{L_{0}}^{L}\left|v_{x}^{m}\right|^{2} d x\right]^{\frac{p_{2}-1}{2}}\left|v_{x t}^{m}\right|\left|v_{t t}^{m}\right|
$$

Taking into account the first estimate $(3,13),(3,15),(3,17)$, from $(3,14)$ and the Gronwall inequality we conclude that

$$
E_{2}\left(t, u^{m}, v^{m}\right) \leq c
$$

which imply that

$$
\begin{aligned}
& \left\{u_{t}^{m}, v_{t}^{m}\right\} \rightarrow\left\{u_{t}, v_{t}\right\} \text { weakly * in } L^{\infty}\left(0, T ; H^{1}\left(0, L_{0}\right) \times H^{1}\left(L_{0}, L\right)\right) \\
& \left\{u_{t t}^{m}, v_{t t}^{m}\right\} \rightarrow\left\{u_{t}, v_{t}\right\} \text { weakly * in } L^{\infty}\left(0, T ; L^{2}\left(0, L_{0}\right) \times L^{2}\left(L_{0}, L\right)\right)
\end{aligned}
$$


Therefore we have $\{u, v\}$ satisfies $(1,1)-(1,5)$ and we have

$$
\mid \begin{aligned}
& -b u_{x x}=-\rho_{1} u_{t t}+\mu_{1} f_{1}(u) \in L^{2}\left(0, L_{0}\right) \\
& -a(t) v_{x x}=-\rho_{2} v_{t t}-g\left(v_{t}\right)+\mu_{2} f_{2}(v) \in L^{2}\left(L_{0}, L\right) \\
& u\left(L_{0}, t\right)=v\left(L_{0}, t\right), \quad b u_{x}\left(L_{0}, t\right)=a(t) v_{x}\left(L_{0}, t\right) \\
& u(0, t)=0=v(L, t)
\end{aligned}
$$

then using lemma 3.2 we have the required regularity to $\{u, v\}$

\section{Exponential Decay}

In this section we study the asymptotic behavior of the solution of system $(1,1)-(1,7)$. In the remainder of this paper we denote by $c$ a positive constant which takes we shall suppose that

$$
\begin{gathered}
\rho_{1} \leq \rho_{2} \quad \text { and } \\
\left.a(t) \leq b, \quad a_{t}(t) \leq 0, \quad \forall t \in\right] 0, \infty[
\end{gathered}
$$

\section{Theorem 4.1}

then there exists positive constants $\gamma$ and $c$ such that

$$
E(t) \leq c E(0) e^{-\gamma t} \quad \forall t \geq 0 .
$$

We shall prove this theorem for strong solutions; our conclusion follow by standard density arguments.

The dissipative property of system $(1,1)-(1,7)$ is given by the following lemma.

\section{Lemma 4.2}

The first order energy satisfies

$$
\frac{d}{d t} E_{1}(t, u, v)=-\left(g\left(v_{t}\right), v_{t}\right)+a_{t}\left|v_{x}\right|^{2}
$$

Proof

Multiplying equation $(1,1)$ by $u_{t}$, equation $(1,2)$ by $v_{t}$ and performing an integration by parts we get the result.

Let $\psi \in C_{0}^{\infty}(0, L)$ be such that $\psi=1$ in $] L_{0}-\delta, L_{0}+\delta[$ for some $\delta>0$, small constant. Let us introduce the following functional

$$
I(t)=\int_{0}^{L_{0}} \rho_{1} u_{t} q u_{x} d x+\int_{L_{0}}^{L} \rho_{2} v_{t} \psi q v_{x} d x
$$


where $q(x)=x$.

Lemma 4.2 There exists $c_{1}$ such that

$$
\begin{gathered}
\frac{d}{d t} I(t) \leq-\frac{L_{0}}{2}\left\{\left(\rho_{2}-\rho_{1}\right) v_{t}^{2}\left(L_{0}, t\right)+a(t)\left[1-\frac{a(t)}{b}\right]\right\} \\
-L_{0}\left[F_{2}\left(v\left(L_{0}, t\right)\right)-F_{1}\left(u\left(L_{0}, t\right)\right)\right]-\frac{1}{2} \int_{0}^{L_{0}}\left(\rho_{1} v_{t}^{2}+b v_{x}^{2}-2 \mu_{1} F_{1}(u)\right) d x \\
-\frac{a(t)}{4} \int_{L_{0}}^{L} v_{x}^{2} d x+c_{1}\left\{\int_{L_{0}+\delta}^{L}\left(v_{t}^{2}+v_{x}^{2}\right) d x+\int_{L_{0}}^{L} g\left(v_{t}\right)^{2} d x+|v|_{p_{2}+1}^{p_{2}+1}\right\}
\end{gathered}
$$

Proof

Multiplying equation $(1,1)$ by $q u_{x}$, equation $(1,2)$ by $\psi q v_{x}$, integrating by parts and using the corresponding boundary conditions, we have

$$
\begin{gathered}
\frac{d}{d t}\left(\rho_{1} u_{t}, q \psi u_{x}\right)=\frac{L_{0}}{2}\left[\rho_{1} u_{t}^{2}\left(L_{0}, t\right)+b u_{x}^{2}\left(L_{0}, t\right)\right]+L_{0} F_{1}\left(u\left(L_{1}, t\right)\right) \\
-\frac{1}{2} \int_{0}^{L_{0}}\left[\rho_{1} u_{t}^{2}+b u_{x}^{2} \cdot-2 F_{1}(u)\right] d x \\
\frac{d}{d x}\left(\rho_{2} v_{t}, q \psi v_{x}\right) \leq-\frac{L_{0}}{2}\left[\rho_{2} v_{t}^{2}\left(L_{0}, t\right)+a(t) v_{x}^{2}\left(L_{0}, t\right)-L_{0} F_{2}\left(v\left(L_{0}, t\right)\right)\right] \\
-\frac{a(t)}{4} \int_{L_{0}}^{L_{0}+\delta} v_{x}^{2} d x+c_{1}\left[\int_{L_{0}+\delta}^{L}\left(v_{t}^{2}+v_{x}^{2}\right) d x+\int_{L_{0}}^{L}\left(g\left(v_{t}\right)^{2}+\left|F_{2}(v)\right|_{\mathbb{R}}\right) d x\right]
\end{gathered}
$$

Summing up $(4,5)$ with $(4,7)$ our conclusion follows.

Let $\varphi \in C^{\infty}(\mathbb{R})$ a nonnegative function such that $\varphi=0$ in $\left.I_{\delta / 2}=\right] L_{0}-\frac{\delta}{2}, L_{0}+\frac{\delta}{2}[$ and $\varphi=1$ in $\mathbb{R} \backslash I_{\delta}$ and consider the functional

$$
J(t)=\int_{L_{0}}^{L} \rho_{2} v_{t} \varphi v d x
$$

We have the following lemma

Lemma 4.4

Given $\varepsilon>0$, there exists a positive constant $c_{\varepsilon}$ such that

$$
\frac{d}{d t} J(t) \leq-\frac{a(t)}{2} \int_{L_{0}+\delta}^{L} v_{x}^{2} d x+\varepsilon a(t) \int_{L}^{L_{0}+\delta} v_{x}^{2} d x+c_{\varepsilon} \int_{L_{0}}^{L}\left(v_{t}^{2}+g\left(v_{t}\right)^{2}\right) d x+|v|_{p_{2}+1}^{p_{2}+1}
$$




\section{Proof}

Multiplying equation $(1,2)$ by $\varphi v$ and integrating by parts we get

$$
\begin{aligned}
\frac{d}{d t} J(t)= & -a(t)\left(v_{x}, \varphi v_{x}\right)-a(t)\left(v_{x}, \varphi_{x} v\right)+\rho_{2}\left(v_{t}, \varphi v_{t}\right)-\left(g\left(v_{t}\right), \varphi v\right) \\
& +\mu_{2}\left(f_{2}(v), \varphi v\right)
\end{aligned}
$$

Applying Young's inequality and hypothesis $(A, 1)$ we conclude our assertion Let us consider the following functional

$$
K(t)=I(t)+\left(2 c_{1}+1\right) J(t)
$$

Using lemma 4.3 and fixing $\varepsilon=\varepsilon_{1}$ in lemma 4.4 , where $\varepsilon_{1}$ is the solution of the equation

$$
\left(2 c_{1}+1\right) \varepsilon_{1}=\frac{1}{8}
$$

we have that there exists a positive constant $c_{2}$ such that

$$
\begin{aligned}
\frac{d}{d t} K(t) \leq & -E_{1}(t, u)-\frac{1}{8} \int_{L_{0}}^{L} a(t) v_{x}^{2} d x+\varepsilon E(t, u, v)+ \\
& c_{2} \int_{L_{0}}^{L}\left(v_{t}^{2}+g\left(v_{t}\right)^{2}+(v)^{p_{2}+1}\right) d x
\end{aligned}
$$

To arrive at the desired inequality on $E(t)$ we must estimate the last term in $(4,8)$, we need the following result.

\section{Lemma 4.5}

Let $\{u, v\}$ be a solution in theorem 3.3.

Then there exist $T_{0}>0$ such that if $T \geq T_{0}$ we have

$$
\begin{aligned}
\int|v|^{p_{2}+1} d x \leq & \varepsilon\left[\int_{s}^{T}\left(b\left|u_{x}\right|^{2}+\left|u_{t}\right|^{2}\right) d s+\int_{s}^{T}\left|a^{1 / 2} v_{x}\right|^{2} d s\right]+ \\
& +c_{\varepsilon} \int_{s}^{T}\left|v_{t}\right|^{2} d s
\end{aligned}
$$

for any $\varepsilon>0$ and $c_{\varepsilon}$ is a constant depending on $T$ and $\varepsilon$, for any initial data $\left\{u^{0}, v^{0}\right\},\left\{u^{1}, v^{1}\right\}$ satisfying $E(0, u, v) \leq k$ where $k>0$ is fixed and $0<S<T<\infty$.

Proof.

The proof is given quite similarly as in the proof of $(4,11)$ in $[8]$ and we will omit it. 


\section{Proof of theorem 4.1.}

From hypothesis on the function $g$, with $p=1$, we get

$$
\int_{L_{0}}^{L}\left(v_{t}^{2}+g\left(v_{t}\right)^{2}\right) d x \leq\left(c_{1}^{-1}+c_{2}\right) \int_{L_{0}}^{L} g\left(v_{t}\right) v_{t} d x
$$

Let us denote

$$
L(t)=N E(t)+k(t)
$$

with $N>0$. Using Young's Inequality and taking $N$ large enough we find that

$$
\theta_{0} E(t) \leq L(t) \leq \theta_{1} E(t)
$$

for some positive constants $\theta_{0}$ and $\theta_{1}$.

Substituting $(4,9),(4,10),(4,11)$ in $(4,8)$, using the identity of lemma 4.2 and integrating from $S$ to $T$, where $0 \leq S<T<\infty$ we obtain

$$
\int_{S}^{T} E(t) d t \leq c E(s)
$$

This inequality, by lemma 2.1 , implies that

$$
E(t) \leq c E(0) e^{-\gamma t}
$$

The proof of theorem 4.1 is completed.

\section{Referencias}

[1] AASSILA M. Strong asymptotic stability of isotropic elasticity system with internal damping. Acta Scientiarium Mathematicarum (Szeged) ; 64:103-108, 1998

[2] DAFERMOS CM. Asymptotic behaviour of solution of evolution equation. In Nonlinear Evolution Equations,Grandall MG (ed.). Academic Press: New York, 103-123, 1978

[3] HARAUX A. Comportement à línfini pour une èquation des ondes non linéaire dissipative.Comptes Rendus Academic Sciences Paris; 287A:507-509, 1978

[4] MARTINEZ P. A new method to obtain decat rate estimates for dissipative systems with localized damping. Revista de Matematicas Complutense ; 12:251-283, 1999

[5] NAKAO M. A difference inequality and its applications to non-linear evolution equations. Journal of the Mathematical Society of Japan; 30:747-762, 1978 
[6] NAKAO M. Energy decay for the wave equation with a non-linear weak disipation. Differential Integral Equations; 8:681-688, 1975

[7] NAKAO M. Decay of solutions of the wave equation with a local non-linear dissipation, Mathematische Annalen; 305:403-417, 1996

[8] WEIJIU LIU and G. WILLIAMS; The exponential stability of the problem of transmission of the wave equation. Bull. Austral. Math. Soc. Vol. 57(1), 305-327, 1998.

[9] MUÑOZ RIVERA J.E. and PEREZ SALVATIERRA A.; Decay of the energy to partially viscoelatic materials. Mathematical models and methods for smart materials, Ser. Adv. Math. Appl. Sci., 62,297-311, 2002.

[10] CAVALCANTI M. M. , V. N. D. CAVALCANTI, J. S. PRATES, J.A. SORIANO; Existence and uniform decay of solutions of a degenerate equation with nonlinear boundary damping and boundary memory source term. Nonlinear Analysis Vol. 38(1), 281-294, 1999.

[11] MUÑOZ RIVERA J. E. and PORTILLO OQUENDO H.; The transmission problem of viscoelastic waves.Acta Aplicandae Mathematicae Vo: 60(1), 1-21, 2000.

[12] MUÑOZ RIVERA J.E. and TO FU M. ; Exponential stability of a transmission problem.To appear.

[13] MUÑOZ RIVERA J.E. and RACKE R. ; Magneto-elasticity-large-time behavior for linear systems.Adv. Differentual Equations, 6(3), 359-384, 2001.

[14] LADYZHENSKAYA O. A. and URAL'TSEVA N. N. ; Linear and Quaslinear Elliptic Equations, Academic Press. New York, 1968.

[15] MUÑOZ RIVERA J.E. and CABANILLAS LAPA E. The nonlinear transmission problem with time dependent coefficients(To appear) 